
\title{
25 Research Square \\ Tick-Borne Encephalitis Affects Sleep-Wake Behavior and Locomotion in Infant Rats
}

\section{Gabriele Chiffi}

University of Bern

Denis Grandgirard

University of Bern

Sabrina Stöckli

University of Bern

Luca G. Valente

University of Bern

Antoine R. Adamantidis

University of Bern

Stephen L. Leib ( $\nabla$ stephen.leib@ifik.unibe.ch )

University of Bern https://orcid.org/0000-0002-1106-6123

\section{Research}

Keywords: Tick-borne encephalitis, Langat Virus, Infant Rats, Sleep, Sleep-wake behavior Cytokines, Chemokines, Neurofilament, Locomotion, Anxiety-like behavior

Posted Date: November 1st, 2021

DOl: https://doi.org/10.21203/rs.3.rs-968597/v1

License: (1) (1) This work is licensed under a Creative Commons Attribution 4.0 International License.

Read Full License 


\section{Abstract}

Background/Aims: Tick-borne encephalitis (TBE) is a disease affecting the central nervous system. Over the last decade, the incidence of TBE has steadily increased in Europe and Asia despite the availably of effective vaccines. Up to $50 \%$ of patients after TBE suffer from post-encephalitic syndrome that may develop into long-lasting morbidity. Altered sleep-wake functions have been reported by patients after TBE. The mechanisms causing these disorders in TBE are largely unknown to date. As a first step toward a better understanding of the pathology of TBEV-inducing sleep dysfunctions, we assessed parameters of sleep structure in an established infant rat model of TBE.

Methods: 13-day old Wistar rats were infected with $1 \times 10^{6} \mathrm{FFU}$ Langat virus (LGTV). On day 4, 9, and 21 post infection, Rotarod (balance and motor coordination) and open field tests (general locomotor activity) were performed and brains from representative animals were collected in each subgroup. On day 28 the animals were implanted with a telemetric EEG/EMG system. Sleep recording was continuously performed for 24 consecutive hours starting at day 38 post infection and visually scored for Wake, NREM, and REM in 4 seconds epochs.

Results: As a novelty of this study, infected animals showed a significant larger percentage of time spend awake during the dark phase and less NREM and REM compared to the control animals $(p<0.01$ for all comparisons). Furthermore, it was seen, that during the dark phase the wake bout length in infected animals was prolonged $(p=0.043)$ and the fragmentation index decreased $(p=0.0085)$ in comparison to the control animals. LGTV-infected animals additionally showed a reduced rotarod performance ability at day $4(p=0.0011)$ and day $9(p=0.0055)$ and day $21(p=0.0037)$. A lower locomotor activity was also seen at day $4(p=0.0196)$ and day $9(p=0.0473)$.

Conclusion: Our data show that experimental TBE in infant rats affects sleep-wake behavior, leads to decreased spontaneous locomotor activity, and impaired moto-coordinative function.

\section{Background}

Tick-borne encephalitis virus (TBEV) is a positive sense single stranded RNA virus that belongs to the Flaviviridae family causing tick-borne encephalitis (TBE) [1]. Transmission of TBEV is typically through the bite of an infected Ixodes tick [2]. Alternative modes of transmission, through ingestion of unpasteurized milk, can also occur $[3,4]$. Three different TBEV subtypes have been identified, namely the European (TBEV-Eur), Siberian (TBEV-Sib), and Far Eastern (TBEV-FE) [2].

Although efficacious vaccines are available, over the last years, TBE has become a growing public health problem in Europe and Asia [5,6]. The number of human cases of TBE has increased by $400 \%$ over the last 30 years [7].

Infection with TBEV-Eur produces a characteristic biphasic disease course. After a short incubation time usually lasting from 2 to 4 days, the first phase occurs (viremia). During the viremic phase of the illness, 
influenza-like symptoms are observed. Symptoms resolve after 2-6 days. After an asymptomatic interval of approximately 7 days, $45-65 \%$ of patients develop neurological signs and symptoms due to infection of the central nervous system (CNS) [8,9]. The infection of the CNS can manifest in inflammation of the meninges (meningitis), the brain parenchyma (encephalitis), the spinal cord (myelitis), the nerve roots (radiculitis), alone or in combination. In contrast to the TBEV-Eur, the Eastern TBEV subtypes (TBEV-Sib and TBEV-FE) are predominantly monophasic $[8,9]$. While the TBEV-FE shows the highest mortality of up to $30 \%$, the TBEV-Eur and TBEV-Sib have much lower mortality rates from $1-2 \%$ and $6-8 \%$ respectively [2]. There is no specific antiviral treatment available for TBE. Patients often require in-hospital treatment for supportive care depending on the severity of signs/symptoms $[4,10]$.

TBE may cause long-lasting morbidity, named post-encephalitic syndrome, which significantly affects daily activities and the quality of life. Thirty to $50 \%$ of patients after acute TBE develop a postencephalitic syndrome. Several neurological/neuropsychiatric symptoms have been documented in different prospective and retrospective studies. Most often, cognitive disorders, neuropsychiatric complaints (apathy, irritability, memory, and concentration disorders) as well as sleep-wake disorders are reported, such as altered sleep patterns or fatigue. Furthermore, headache, hearing loss with or without tinnitus, vision disturbances, balance as well as coordination disorders, and flaccid paresis or paralysis are also reported $[4,11-16]$.

A wide variety of sleep-wake disorders has been reported during the acute phase of the TBE infection as well as during short and long-term follow-ups [17-19]. Unfortunately, only few studies used clear definitions of sleep-wake disorders or assessed them using objective measurement tools or standardized questionnaires. Furthermore, sleep-wake disorders after TBE may well be under-diagnosed or underreported [14]. However, several studies have shown, that TBE patients suffer significantly more frequently from daytime fatigue compared to age-matched controls $[18,19]$. Additionally, newly occurring narcolepsy after the TBE vaccination has been reported [20].

With this study, we aimed to investigate sleep-wake patterns in an established model of TBE using infant rats infected with Langat Virus (LGTV).

\section{Materials And Methods}

\section{Animals}

All animal studies were approved by the Animal Care and Experimentation Committee of the Canton Bern, Switzerland, and Swiss national regulation for animal protection (License number BE 88/18). A wellestablished tick-borne encephalitis infant rat model was used [21]. Suckling Wistar strain pups (8 -dayold rats with the mothers at the time of supply, Charles River Laboratories, Sulzfeld, Germany) were used in the experiments. Overall, 11 litters were used in the study, containing 113 pups. 58 pups were included in the infection group and 55 in the control group.

\section{Virus production}


The LGTV strain TP21 was kindly provided by Dr. Daniel Růžek (Department of Virology, Veterinary Research Institute, Brno, Czech Republic), and the stocks were prepared by amplification in PC12 rat pheochromocytoma cells for 7 days. The virus stock was then concentrated from culture supernatant by ultrafiltration using an Amicon ultra-15, PLHK Ultracel-PL Membran, 100 kDa (Milipore AG, Zug, Switzerland). The quantification of the virus concentration was done using an immunoperoxidase focus assay, as previously described [22]

\section{Infection of pups}

After an acclimatization period of 5 days, rat pups were infected by intracisternal injection of 1 x $10^{6} \mathrm{FFU}$ of the Langat Virus in a volume of $25 \mu \mathrm{L}$. As a sham procedure, animals were injected with $25 \mu \mathrm{L}$ of the PC12 cell medium. The intracisternal injections were performed by using a BD Micro-fine ${ }^{\text {TM }} 0.3 \mathrm{~mL}$ syringe. The injection site was disinfected before injecting the fluid and it was made sure, that the needle was not inserted more than $3 \mathrm{~mm}$. The animals were kept under standard post-op care, consisting of a 2hour close monitoring period after the injection and daily wellness checks afterward.

\section{Behavioral tests}

Rotarod test: Animals were subjected to the Rotarod test at day 4, 9, and 21 post infection (pi), where the ability to stay on a rotation drum (Cat. No. 47700, Ugo Basile srl, Comerio, Italy) was tested. The rotating drum was started at a speed of 5 revolutions per minute and accelerated up to 40 rotations per minute over 5 minutes. The rats were placed individually on the drum and once they are stable, the acceleration started. Latency to fall was then determined. Each animal performed the test three times, and the average time of all three trials was used for the analysis [21].

Open Field test: The open field test was performed at day 4, 9, and 21 pi. Animals were placed in the middle of a dimly lit arena ( $45 \times 45 \times 40 \mathrm{~cm})$ for 2 minutes. The total walking distance (in centimeters), and the time spent in the center/periphery were determined for each animal using a video tracking system (Noldus EthoVision $\mathrm{XT}$ ). Each animal repeated the test three times at every time point.

\section{CSF, Blood and Brain sampling}

CSF sampling was performed on days 4,9 , and 21 pi by puncturing the cisterna magna. Depending on the body weight of the animal between $15-30 \mu$ of sample volume was withdrawn. The CSF was centrifuged at $13000 \mathrm{rpm}$ for $10 \mathrm{~min}$ at $4^{\circ} \mathrm{C}$ using a table-top centrifuge and the supernatant was transferred into a new tube and stored at $-80^{\circ} \mathrm{C}$ until further analysis.

At days 4, 9, and 21 pi, a subset of the animals was sacrificed by intraperitoneal injection of pentobarbital (Esconarkon®, 150 mg/kg, Streuli Pharma AG, Switzerland) for blood and brain sampling.

For the blood sampling, blood was collected from the right ventricle, which was accessed via thoracotomy. Blood was collected using a 23-gauge needle, and $0.5-1.0 \mathrm{~mL}$ were collected. Blood samples were placed at room temperature for 30 minutes enabling clotting. After 30 minutes the samples 
were centrifuged in a refrigerated centrifuge $\left(4^{\circ} \mathrm{C}\right)$ for 10 min at $2000 \mathrm{x} \mathrm{g}$ and serum was collected and transferred into a new tube and stored at $-80^{\circ} \mathrm{C}$ until further processing.

To harvest the brains, animals were perfused with 25-30 mL ice-cold PBS using a 25-gauge butterfly needle, inserted into the left cardiac ventricle, while an incision was made at the right atrium. The brain was then removed and placed on dry ice and stored at $-80^{\circ} \mathrm{C}$.

\section{Implantation of the EEG and EMG electrodes}

The telemetry EEG and EMG electrodes were implanted following the manufacturer's instructions (Figure 1). Animals were anesthetized with isoflurane in oxygen (5\% for induction, $1.5-2.5 \%$ for maintenance). Under deep anesthesia abdomen, neck, and head of the animal were shaved. On the shaved sites Betadine ${ }^{\circledR}$ was applied to avoid postoperative infection and Lidocaine (Lidocain $\mathrm{HCl}$ "Bichsel" 0.5\%, $5 \mathrm{mg} / \mathrm{kg}$ ) was applied locally subcutaneous (s.c.) at the site of the incision before surgery. Under aseptic conditions, the telemeter body (Kaha Sciences, TR50BB) was implanted in the abdominal cavity and secured to the abdominal wall using the suture tabs on the telemeter body (Figure 1A). Once the telemeter body was secured the abdominal wall was closed with sutures ("Silk", black silk 1,5 (4/0), 100m, Dieckhoff \& Ratschow Praxisdienst GmbH \&Co.KG, Germany). A subcutaneous blunt dissection was performed to the nape of the neck followed by a small incision. The electrode leads were then threaded through the trocar and externalized at the neck. Before implanting the EMG electrodes, blunt dissection to expose the nuchal muscle was performed. The EMG electrodes were secured in place on the ipsilateral nuchal muscle with a suture with their extremities placed into the muscle (Figure 1B).

A midline incision through the scalp and clean connective tissue from the skull was performed. Holes were drilled using a 0.9-mm steel drill (1RF 009, Meisinger, Germany). One electrode was implanted in the dura mater of the parietal cortex ( $2 \mathrm{~mm}$ posterior of the bregma and $4.5 \mathrm{~mm}$ lateral to the midline in the right), and the second in the cerebellar cortex ( $2 \mathrm{~mm}$ posterior of the lambda and $2 \mathrm{~mm}$ lateral to the midline in the right) as described in [23] (Figure 1C). For securing the electrodes dental cement (Vertex ${ }^{\text {TM }}$ Self Curing number 3, 1000gr; Vertex Self Curing liquid 1000mL, ITD Intertrading Dental AG, Switzerland) was applied on the skull. As an anchor for the dental cement two screws (1.1 mm O.D. x 1/8 inch, F00CE125, J.I. Morris) were implanted in the skull.

After implantation, incisions were closed using wound clips (Autoclip Wound Closing System - Clips $9 \mathrm{~mm}$ ). Postoperative analgesia was provided after surgery and for the three following days (Metacam® 5 $\mathrm{mg} / \mathrm{kg}$, daily, s.c., Boehringer Ingelheim Vetmedica $\mathrm{GmbH}$, Germany) and animals were monitored twice a day until EEG measurement.

\section{EEG Measurement and scoring}

EEG and EMG from each animal were measured for 24 consecutive hours on day 39 p.i. (Figure 1D), with two animals co-housed in a cage. A previously published algorithm was used for automatic scoring [24]. 
The scoring was then visually checked for correctness and to score epochs which were classified as indetermined by the algorithm.

The visual check was done using an open-source tool for visual EEG analysis with some adaptations [25]. To facilitate the visual analysis, the collected data was band-pass filtered using a Butterworth filter with the cutoffs set at $0.5 \mathrm{~Hz}$ and $40 \mathrm{~Hz}$ respectively.

Based on sleep scoring, the macrostructure of the sleep was determined for a full day period, as well as for the light and the dark period separately. The bout number and the average bout duration of each sleep stage were also calculated. Lastly, the delta power during NREM and the theta power during REM were calculated as a marker of the microarchitecture of the sleep-wake behavior.

\section{RNA extraction}

Brain hemispheres were divided into cerebellum, midbrain, and frontal sections. The brain tissue was placed in the appropriate volume of TRIzol® Reagent ( $1 \mathrm{~mL}$ per $100 \mathrm{mg}$ tissue, Invitrogen) and homogenized (TissueRuptor II, Qiagen) for 20 seconds at full speed. RNA extraction was performed using an RNA Tissue Kit (PureLink RNA extraction kit, ThermoFischer; Universal RNA Tissue Kit, Qiagen) following the manufacturer's instructions. The RNA was eluted with $2 \times 40$ ul of RNase free water. The quality and quantity of the extracted RNA were assessed with NanoDrop ${ }^{\mathrm{TM}}$ One $\mathrm{C}$ (Thermo Scientific ${ }^{\mathrm{TM}}$ ).

\section{Primers}

The primers which were used for the detection of LGTV strain TP21 were already described by [21] and were purchased from Microsynth (Balgach, Switzerland). LGTV forward primer 5'-

TGTGTGGAGCGGCGATT-3', LGTV reverse primer 5'-TAAGGGCGCGTTCCATCTC-3' and LGTV probe 5'(FAM)-CTTGGCCCCCACACGAGTGGTG-(BHQ-1)-3' were used.

\section{One-Step Real-time PCR}

RT-PCR was performed according to the manufactures instructions and as described in a total reaction volume of $25 \mu \mathrm{l}[21,26]$ on a QuantStudio ${ }^{\text {TM }} 6$ Flex System with the QuantStudio ${ }^{\text {TM }}$ Real-Time PCR software (Applied Biosystems). The following parameters were used: reverse transcription at $50^{\circ} \mathrm{C}$ for 10 minutes, polymerase activation at $95^{\circ} \mathrm{C}$ for 5 minutes, 40 cycles of amplification with a two-step cycling of 3 seconds at $95^{\circ} \mathrm{C}$ and 30 seconds at $60^{\circ} \mathrm{C}$. To determine the amount of virus particles in the brain parenchyma, a standard curve with a 10 -fold serial dilution of a virus vial with known concentration was used.

\section{Cytokine and Chemokine in the CSF and Serum}

Five cytokines (IL-6, IFN- $\gamma$, MCP-1, IP-10, and RANTES) were assessed in the CSF and in the blood serum using a magnetic multiplex assay (MILLIPLEX MAP Rat Cytokine/Chemokine Magnetic Bead Panel Immunology Multiplex Assay, Millipore) on a Bio-Plex 200 station (Bio-Rad Laboratories). For the three 
timepoints (day 4, day 9, and day $21 \mathrm{pi}) 10 \mu \mathrm{l}$ of CSF was diluted to a $50 \mathrm{ul}$ final volume. For statistical purposes, values for the samples below the detection limit were calculated using the detection limit provided by the manufacturer multiplied by the dilution factor (IL- $630.7 \mathrm{pg} / \mathrm{mL}$, IFN-y $6.2 \mathrm{pg} / \mathrm{mL}, \mathrm{MCP}-1$ $9.0 \mathrm{pg} / \mathrm{mL}, \mathrm{IP}-101.4 \mathrm{pg} / \mathrm{mL}$, RANTES $1.3 \mathrm{pg} / \mathrm{mL}$ )

\section{Neurofilament in the CSF}

Neurofilament light chain (NfL) in the CSF at different timepoints (day 4, 9, and 21 pi) was analyzed using an automated ELISA-based microfluidic system (ELLA, ProteinSimple, San Jose, CA, USA) with dedicated cartridges according to the manufacturer instructions, as previously described [27].

\section{Statistical analysis}

The distribution of the data was visually assessed and based on the Shapiro-Wilk test. Data was not normally distributed and therefore analyzed using the nonparametric Mann-Whitney $\mathrm{U}$ test. One-tailed tests were performed when pre-existing one-tailed hypotheses based on a previous publication of our group [21] could be expected. Sleep analyses were conducted without pre-conceived hypotheses, using a two-tailed set-up. For the analysis of the three-hour bins, the comparisons were done using ANOVA. Values of $p<0.05$ were considered as statistically significant. All of the statistical analyses were conducted using R-Studio, while the plots were done using Matlab 2019b.

\section{Results}

\section{Confirmation of LGTV infection}

The infection with the Langat virus was confirmed by one-step real-time PCR (Figure 2A and Table 1). Four days after infection, animals showed the highest number of RNA copies in the cerebellum, followed by the forebrain and the midbrain. A similar pattern was observed at day 9 , at day 21 post infection, and after sleep recording (day 42 post infection).

Overall, the number of RNA copies steadily declined over the investigated time points. For the control animals, no viral RNA was detected.

No difference in the weight or the weight gain could be observed between the control animals and the infected animals, as depicted in an additional figure [see additional figure 1]. Survival was $100 \%$, in both uninfected and infected animal groups. 
Table 1: Number of LGTV RNA copies: RNA copies measured at Day 4, Day 9, Day 21, and after the sleep measurement.

\begin{tabular}{cccc}
\hline & $\begin{array}{c}\text { Cerebellum } \\
\text { [copies/g tissue] }\end{array}$ & $\begin{array}{c}\text { Midbrain } \\
\text { [copies } / \mathrm{g} \text { tissue] }\end{array}$ & $\begin{array}{c}\text { Forebrain } \\
\text { [copies/g tissue] }\end{array}$ \\
\hline Day 4 & $4^{\prime} 300^{\prime} 000$ & $270^{\prime} 000$ & $960^{\prime} 000$ \\
\hline Day 9 & $560^{\prime} 000$ & $33^{\prime} 000$ & $44^{\prime} 000$ \\
\hline Day 21 & $34^{\prime} 000$ & $4^{\prime} 600$ & $5^{\prime} 200$ \\
\hline After Sleep & $1^{\prime} 770$ & $1^{\prime} 140$ & $1^{\prime} 110$ \\
\hline
\end{tabular}

\section{CSF parameters}

Chemokines, cytokines and Neurofilament light chain (NfL), were assessed in the CSF of the animals at day 4 , day 9 , and day 21 pi. (Figure 2B - $\mathbf{G}$ and Table 2). The CSF levels of RANTES and IP-10 were significantly higher in infected animals compared to control animals at day 4 , day 9 , and day 21 pi (Figure 2B and C). At day 4 pi IL-6 and IFN- $y$ were significantly higher in infected animals (Figure 2D and E). Significant differences were also observed for MCP-1 at day 4 and day 9 pi (Figure 2F).

CSF levels of NfL were similar between infected and controls animals at day 4 (401.47 \pm 67.7 vs $472.77 \pm$ $106.6 \mathrm{pg} / \mathrm{mL})$ and $21 \mathrm{pi}(392.7 \pm 58.0 \mathrm{vs} 346.6 \pm 59.2 \mathrm{pg} / \mathrm{mL})$. At day 9 (Figure 2G), a significant $(p=0.0083)$ increase of NfL levels in the CSF was observed in infected animals $(1065.2 \pm 288.6 \mathrm{pg} / \mathrm{mL})$ compared to the control animals $(633.5 \pm 353.1 \mathrm{pg} / \mathrm{mL})$.

\section{Table 2: CSF concentrations of inflammatory parameters and NfL.}

\section{Blood serum parameters}

The cytokines, chemokines, and NfL assessed in the CSF were also assessed in the blood serum. No significant difference between the two groups could be observed as it can be seen in an additional table [see additional table 1].

\section{Behavioral tests}

In infected animals, a durable impairment in the Rotarod test was documented (Figure 3A). A shorter latency to fall was determined in LGTV-infected animals compared to control animals at 4 days pi (39.31 \pm 3.04 s vs. $51.47 \pm 3.36 \mathrm{~s}, p=0.0011)$, at 9 days $p i(91.69 \pm 15.50 \mathrm{~s}$ vs. $138.89 \pm 15.77 \mathrm{~s}, p=0.0055)$ and at 21 days pi (103.43 $\pm 16.26 \mathrm{~s}$ vs. $172.04 \pm 17.11 \mathrm{~s}, \mathrm{p}=0.0037)$.

In the open field test, two parameters were determined. General locomotor activity was assessed by determining total walking distance. Infected animals moved less than the control animals. This resulted in a significant difference at day $4(502.0 \pm 2.9 \mathrm{~cm}$ vs. $579.7 \pm 3.1 \mathrm{~cm}, \mathrm{p}=0.0196)$ and day $9 \mathrm{pi}(584.9 \pm$ $2.6 \mathrm{~cm}$ vs. $631.5 \pm 2.6 \mathrm{~cm}, \mathrm{p}=0.0473)$ and a trend at day $21 \mathrm{pi}(605.7 \pm 5.7 \mathrm{~cm}$ vs. $656.9 \pm 4.3 \mathrm{~cm}, \mathrm{p}=$ 0.0646 , Figure 3B).

Furthermore, the exploratory behavior of the animals was assessed by determining the time spent in the center and the periphery of the arena (Figure $3 \mathrm{C}$ ). Control animals spent significantly more time in the 


\begin{tabular}{|c|c|c|c|c|c|}
\hline Cytokine & Timepoint & Control $[\mathrm{pg} / \mathrm{mL}]$ & Infection [pg/mL] & p-Value & of the arena \\
\hline \multirow[t]{3}{*}{ RANTES } & Day 4 & $16.79(n=14)$ & $503.18(n=20)$ & 0.0033 & $\begin{array}{l}\text { compared to Infected } \\
\text { animals at day } 4(6.28 \pm\end{array}$ \\
\hline & Day 9 & $14.54(n=13)$ & $80.31(n=23)$ & 0.0062 & $0.10 \%$ vs. $11.44 \pm 0.19 \%, p$ \\
\hline & Day 21 & $23.98(n=13)$ & $45.39(n=18)$ & 0.0216 & $=0.0123)$ and day $9 \mathrm{pi}(9.96$ \\
\hline \multirow[t]{3}{*}{ IL-6 } & Day 4 & $236.94(n=15)$ & $1363.22(n=26)$ & 0.0082 & $p=0.0101)$ \\
\hline & Day 9 & $145.73(n=15)$ & $142.99(n=28)$ & 0.1816 & alvsis of sleer \\
\hline & Day 21 & $131.69(n=13)$ & $120.02(n=18)$ & 0.6472 & \\
\hline \multirow[t]{3}{*}{ IFN-Y } & Day 4 & $68.13(n=15)$ & $361.22(n=26)$ & 0.0281 & $\begin{array}{l}\text { Sleep macrostructure } \\
\text { (percentage of time spent in }\end{array}$ \\
\hline & Day 9 & $39.68(n=15)$ & $63.23(n=28)$ & 0.1396 & wake, REM, and NREM \\
\hline & Day 21 & $49.07(n=13)$ & $22.25(n=18)$ & 0.7477 & was assessed over \\
\hline \multirow[t]{3}{*}{ MCP-1 } & Day 4 & $224.61(n=15)$ & $697.12(n=26)$ & 0.0164 & sub-divided into the light \\
\hline & Day 9 & $29.04(n=14)$ & $306.09(n=28)$ & 0.0076 & and dark periods (Figure 4A \\
\hline & Day 21 & $204.48(n=13)$ & $460.53(n=18)$ & 0.5255 & and 4B, respectively). \\
\hline \multirow[t]{3}{*}{ IP-10 } & Day 4 & $140.57(n=14)$ & $2188.54(n=22)$ & 0.0001 & When assessing the total \\
\hline & Day 9 & $131.62(n=14)$ & $1533.96(n=26)$ & 0.0003 & $\begin{array}{l}\text { light period, infected } \\
\text { animals spent significantly }\end{array}$ \\
\hline & Day 21 & $279.62(n=13)$ & $1095.21(n=18)$ & 0.0034 & more time awake $(26.74 \%$ \\
\hline \multirow[t]{3}{*}{ NfL } & Day 4 & $472.77(n=13)$ & $401.47(n=19)$ & 0.878 & for the infection and $19.17 \%$ \\
\hline & Day 9 & $633.50(n=14)$ & $1065.21(n=19)$ & 0.0083 & $\begin{array}{l}\text { Tor the control, } p=0.01) \text { and } \\
\text { less in REM sleep }(19.83 \%\end{array}$ \\
\hline & Day 21 & $346.9(n=10)$ & $392.7(n=10)$ & 0.6232 & vs. $26.83 \%, p=0.0006$ ) \\
\hline
\end{tabular}

animals, while NREM sleep was similar between the groups. During the dark period infection animals spent significantly more time awake $(62.70 \%$ vs. $49.85 \%, p=0.00002)$ and significantly less time in $\operatorname{NREM}(29.50 \%$ vs. $37.00 \%, p=0.0047)$ as well as in REM sleep (7.80\% vs. $13.14 \%, p=0.0007$, Figure 4 C).

The differences in the different sleep stages were then assessed in 3 hours bins (Figure 4D-F and Table 3). A significant difference in the fragmentation of the sleep, as determined by the number of state changes, could be documented during the dark period, where the infected animals displayed a lower number of state changes than the control animals (776.5 \pm 71.6 vs $1221.1 \pm 112.4, p=0.0085$, Figure 4G). This was not observed during the light phase.

The lower number of state changes in infected animals was accompanied by a longer duration of the wake bouts in the infected animals during the dark phase as compared to control animals $(1.83 \pm 0.29$ min vs $1.02 \pm 0.23 \mathrm{~min}, p=0.0434$, Figure $4 \mathrm{H}$ ), while no significant difference in NREM and REM bout durations could be detected as depicted in an additional figure [see additional figure 2]. 
Table 3: Sleep macrostructure for 3-hour windows, $n_{\text {contral }}=8, n_{\text {infection }}=10$

\begin{tabular}{|c|c|c|c|c|c|c|c|c|c|}
\hline & \multicolumn{3}{|c|}{ Wake } & \multicolumn{3}{|c|}{ NREM } & \multicolumn{3}{|c|}{ REM } \\
\hline & Control & Infection & p-value & Control & Infection & p-value & Control & Infection & p-value \\
\hline $06: 00-09: 00$ & $9.19 \%$ & $16.27 \%$ & 0.08 & $63.56 \%$ & $63.14 \%$ & 0.92 & $27.25 \%$ & $20.59 \%$ & 0.04 \\
\hline $09: 00-12: 00$ & $27.32 \%$ & $25.94 \%$ & 0.77 & $49.56 \%$ & $53.24 \%$ & 0.31 & $23.11 \%$ & $20.82 \%$ & 0.50 \\
\hline $12: 00-15: 00$ & $14.85 \%$ & $27.43 \%$ & 0.01 & $56.05 \%$ & $53.43 \%$ & 0.50 & $29.11 \%$ & $19.14 \%$ & 0.01 \\
\hline $15: 00-18: 00$ & $25.34 \%$ & $37.31 \%$ & 0.04 & $46.83 \%$ & $43.94 \%$ & 0.55 & $27.83 \%$ & $18.75 \%$ & 0.03 \\
\hline $18: 00-21: 00$ & $52.19 \%$ & $66.42 \%$ & 0.03 & $33.75 \%$ & $25.31 \%$ & 0.06 & $14.07 \%$ & $8.27 \%$ & 0.09 \\
\hline $21: 00-24: 00$ & $46.44 \%$ & $50.34 \%$ & 0.51 & $38.25 \%$ & $38.90 \%$ & 0.89 & $15.31 \%$ & $10.76 \%$ & 0.07 \\
\hline $00: 00-03: 00$ & $50.56 \%$ & $63.79 \%$ & 0.04 & $37.29 \%$ & $29.09 \%$ & 0.09 & $12.15 \%$ & $7.12 \%$ & 0.07 \\
\hline $03: 00-06: 00$ & $50.23 \%$ & $70.23 \%$ & 0.00 & $38.74 \%$ & $24.71 \%$ & 0.01 & $11.04 \%$ & $5.06 \%$ & 0.01 \\
\hline
\end{tabular}

\section{Correlation between sleep and behavior}

A strong correlation could be observed between the last trial of the Rotarod at Day 21 and sleep parameters in the group of infected animals (Figure 4I). As such significant correlations between the Rotarod performance and Wake, NREM in both lightning conditions were observed. Wake was negatively correlated with the latency to fall ( $r=-0.77, p=0.009$ for Light and $r=-0.83, p=0.003$ for Dark), while NREM was positively correlated $(r=0.84, p=0.002$ for Light and $r=0.90, p=0.0003)$. Furthermore, a positive correlation was found between the last trial of the Rotarod at Day 21 and the sleep fragmentation during dark $(r=0.78, p=0.007)$ as well as a negative correlation with the wake bout length during dark $(r=-0.92, p=0.0002)$.

\section{Microstructure}

When assessing sleep microstructure, no significant difference could be found for the delta power during NREM between the groups (Figure $4 \mathbf{J}$ ). For theta power during the REM sleep, a significant difference was found between the groups during the dark period $(p=0.00036$, Figure $4 K)$ with a higher delta power measured for the control animals. The same pattern was not seen during the light period $(p=0.354$, Figure 4K).

\section{Discussion}

In the present study, we investigated how LGTV-induced encephalitis influences sleep behavior in juvenile rats. To the best of our knowledge, this is the first study assessing the consequences of neuroinfection caused by LGTV in wildtype rats over such an extensive time, and with such a large sample, while combining inflammatory parameters, behavior results with regards to locomotion and motor coordination and the assessment of sleep-wake behavior.

The major novelty of this study is the identification and characterization of sleep-wake disorders after LGTV infection. Sleep-wake behavior was assessed for 24 consecutive hours, encompassing a full light and dark cycle. We observed that the control animals spent significant less time awake and significantly 
more time in REM sleep in the light period. In the dark period, the control animal spent significantly less time awake and therefore significantly more time in NREM and REM sleep than the infected animals.

Infected animals had a reduced number of state changes during the dark period compared to the control animals. Additionally, the duration of a wake bout in the dark phase was elongated in the infection animals.

After TBEV, the most consistently reported parameter related to sleep-wake disturbance is fatigue, with a significantly higher number of patients complaining from fatigue compared to control groups $[17-19,28]$. Fatigue is assessed throughout the day and therefore represents a sleep-wake disturbance seen during the active phase of the patients [29].

The results in the experimental model are in line with these observations of sleep-wake disturbances in patients during the active phase. Similar to fatigue in patients, sleep-wake disorders in rats are also found during the active phase, namely, during the dark period. It has been debated in patients, whether fatigue is a direct physiological response to TBEV infection or whether this is a more subjective complaint, potentially arising from indirect changes to the daily routine due to the TBEV infection.

Further, the experimental model revealed clear changes in the sleep macrostructure which were measured with objective parameters compared to a matched control group, under identical conditions. Therefore, our study gives a first indication, that the sleep-wake disorders could be a result of the infection itself and not caused by a change of the patient's circumstances. In patients, there is limited data that support a direct effect of TBEV infection on the sleep-wake disorders. MRI studies from patients show a clustering of virus-induced lesions in the thalamus $[30,31]$. Due to the importance of the thalamus in the induction, stabilization, and termination of sleep [32], the hypothesis, that this affected structure is responsible for sleep-wake disturbance consecutive to TBE, is not unlikely. A prolongation of the wake bouts compared to the controls during the dark phase in the present experimental model further support this hypothesis. Lesion studies targeting the thalamus in experimental models have not yielded substantial changes in the sleep-wake behavior [33]. In thalamic stroke patients, however, a change in the sleep-wake behavior has been reported, including modifications in arousal and in the production of NREM sleep [32,34,35].

Extensive changes in sleep macrostructure, as observed in infected animals, could not be documented in a recent case-control study assessing similar parameters in TBE patients [36]. This discrepancy could be caused by differences in the experimental setup, since the clinical study only conducted measurement during a single night, while data were collected for 24 hours in the experimental study. Further, in animals, the study was conducted at a controlled, constant time, (i.e., within weeks after the infection), while in patients, sleep was assessed on average two years after the infection. Furthermore, even though the cases and controls were similar regarding age, sex, and other factors in the clinical study, the experimental study compared sibling animals, potentially constituting a more appropriate control group. It should additionally be mentioned that, even when the PSG records did not detect significant differences, TBE patients reported statistically more sleep-related functional impairment on the Functional Outcome of Sleep Questionnaire (FOSQ) compared with the controls. 
Changes to the sleep microarchitecture were also observed, with a significant decrease in theta power during REM observed during the dark period in the infected animals. Theta power has been hypothesized to be involved in emotional memory consolidation and processing [37]. Furthermore, it has been seen in other animal models, that theta power is reduced in a tau model of neurodegeneration [38] or in animals that were subjected to chronic social defeat stress [39]. To the best of our knowledge, sleep microarchitecture has never been assessed after tick-borne encephalitis, either experimentally or in patients.

LGTV was detected in the brain parenchyma at each assessed timepoint. At day 4 pi the highest viral load was detected. The viral load decreased over time with the lowest viral load measured after the sleep recording. A similar trend of decrease of viral load over time was seen in previous literature using experimental models $[21,40]$.

Five cytokines were assessed in the CSF and in the serum. In infected animals, IFN- $\gamma$ and IL- 6 levels were significantly higher at day 4 pi than in the control animals. The MCP-1 levels in the CSF were significantly higher in infected animals at day 4 and day 9 pi. The level of IP-10 and RANTES in CSF were significantly higher in infected animals in all three assessed timepoints.

The chemokine and cytokine responses are similar to those that can be observed in patients or other experimental models. RANTES/CCL5 was significantly increased in the CSF of TBE patients at the hospital admission [41,42] as well during as up to 16 days after the admission [41,43]. Induction of RANTES expression was also observed in the brain tissue of infected mice after TBEV infection [44].

In the acute phase of TBE CSF IL- 6 levels were significantly increased in adult TBE patients compared to a control group [45], and was proposed as a useful prognostic tool [46,47]. After 10 to 12 days the IL-6 levels return to levels comparable to those of control patients [45].

Increased CSF levels of IFN- $\gamma$ were also seen in TBE patients with the highest level shortly after the beginning of the infection [48,49]. Furthermore, children with significantly increased IFN- $\gamma$ and IL-6 levels in the acute phase in the CSF developed sequela in the later phase [50].

TBE patients displayed a significant increased level of MCP-1/CCL2 in the CSF at hospital admission $[41,51]$ and a significant increase of MCP-1/CCL2 was also shown in the brain of TBEV infected animals in $[52,53]$.

A significantly increased level of IP-10/CXCL10 in CSF adults and children patients with TBE was described compared to the control group [41] at the admission to the hospital as well as 3 weeks later [54]. The increase of IP-10/CXCL10 over time was shown also in an experimental model, showing significantly increased levels in the brain starting from day 5 post infection [52].

Previous literature has indicated that in children, the extend of the inflammatory response can be predictive of the behavioral impairments. For example, the level of IL- 6 during the acute phase of TBE has been proposed as a predictor for the development of sequelae in children [50]. Data of the present study 
did however not reveal that a cytokine or chemokine can reliably predict behavior with regards to motor coordination as shown in two additional tables [see additional table $\mathbf{2}$ and additional table 3 ].

Sleep disturbances have been previously associated with enhanced cytokine levels of e.g., IL-6 and IP10 in patients $[55,56]$. However, in contrast to these studies in patients, sleep-wake disorders were determined 21 days after infection in the animals of the present study, at a time when most of the cytokines returned to basal levels. It is therefore unlikely that inflammatory is a direct driver of sleep disturbance.

Furthermore, no significant contribution of the IP-10 cytokine levels measured at the earlier time points on the change in sleep-wake behavior could be observed [see additional figure 3 and additional figure 4].

We found a significant increased level of neurofilament light chain (NfL) in the CSF of infected animals at day 9 pi. NfL is a cytoskeletal protein that is found specifically in myelinated subcortical axons. Increased CSF level of $\mathrm{NfL}$ is indicative of axonal damage and neuronal death and has been reported in a wide variety of CNS disorders, such as multiple sclerosis, Parkinson's disease, and amyotrophic lateral sclerosis [57]. Furthermore, an increase of NfL level in the CSF, as well as the serum, has been observed after CNS infections with varicella-zoster virus [58]. It has further been seen in pneumococcal meningitis in an experimental model [59] as well as for childhood bacterial meningitis [60]. To date, CSF levels of $\mathrm{NfL}$ after TBE have been described in one study. The CSF was sampled at a mean duration from neurological symptoms of 4.2 days. TBE patients showed a higher NfL level in the CSF compared to the control indicating a higher level of neuronal injury [61].

When assessing the behavior of the animals, we found, that infected animals showed a significant reduction of balance and motor coordination at all the measurement times.

Human histopathological studies show an affinity of the TBE virus for the basal ganglia, mesencephalon, diencephalon, medulla oblongata, pons, Purkinje cells in the cerebellum as well as the anterior horn cells in the spinal cord $[4,11,62,63]$. In the present study, a high concentration of LGTV was documented in the cerebellum. Since the cerebellum is involved in motor coordination and balance [64], viral infection in this brain area could explain the reduction of locomotion and coordination seen in infected animals. This would be in line with ataxia, often documented in clinical analysis of TBE patients, with an occurrence ranging from $6 \%$ to $72 \%[13,14,16,30,65]$.

In the open field, we saw significant changes in general locomotor activity and the willingness to explore a new environment after the infection. This effect was seen at day 4 and day 9 after the infection. These results are in line with previous results using the same animal model [21]. The open field test is also used to determine the anxiety-like behavior in animals [66]. Infected animals showed anxiety-like behavior with less exploration of the center of the arena. Increased anxiety and depression have been reported in patients in several previous studies $[17,67,68]$. This behavior has not been reported in an animal model of TBE infection, and a previous study even reported reduced anxiety-like behavior in a similar model in wildtype mice after infection [69]. 
A limited number of clinical studies have assessed the relationship between infectious diseases and sleep-wake disorders, and it stays unclear whether changes in sleep-wake behavior could be caused by the virus itself or rather by the immune answer to the infection. Several viral infections, such as Polio, HIV, Hepatitis C, Varicella zoster, and Zika have been associated with the development of sleep-wake disorders. This is best documented for HIV-infected patients, that develop sleep-wake disorders already during the asymptomatic phase of the disease. During HIV, these perturbances are most likely due to a dysregulated immune response rather than caused by the patient's medication $[70,71]$. Using actigraphy, a significant increase in sleep onset latency (SOL) has been observed in people living with HIV compared to a gender- and age-matched control group [72]. Additionally, in a polysomnographic study of people 60 days after a SARS-CoV-2 infection, a mean SOL of approximately 90 minutes was observed [73], which is compared to 20 minutes in age-matched controls [74]. The increase in SOL could be compared to the longer wake bout period which has been observed in our study in the infection group.

A limitation of this study is, that the current EEG/EMG setup did not have a sufficiently high spatial resolution to capture the local aspects of the microarchitecture of sleep e.g., in different brain regions. As such it is difficult to judge how and if the local phenomena of sleep in the infected animals are still maintained or whether these are also disrupted.

As with other studies on long-term outcomes, Langat Virus was selected as a model for TBEV, as TBEV is associated with a high lethality in laboratory rodents $[75,76]$ that precludes the assessment of long-term effects.

\section{Conclusion}

This study extends previous research using the present experimental model and further characterizes features consistent with a post encephalitis syndrome observed in patients after TBE. A reduction of motor coordination and spontaneous locomotion could be documented up to 21 days after infection. Moreover, anxiety-like behavior was observed in the infected animals, a symptom often described in TBE patients.

We also thoroughly characterized sleep-wake behavior after the infection, with significant differences between the infected animals and control animals, mainly during the time rats are active (dark period). This is comparable to the often-reported daytime sleep-wake disorders in TBEV patients, such as fatigue.

However, more research in patients as well as in animals is needed to better understand the underlying causes of sleep-wake disorders after TBE. Moreover, the effect of sleep-wake disorders on the outcome after the TBEV infection should be assessed to evaluate whether treating patients for sleep-wake disorders would be a helpful tool in the neurorehabilitation of these patients.

\section{Abbreviations}


BHQ-1: Black Hole Quencher-1; CNS: Central Nervous System; CSF: Cerebral Spinal Fluid; EEG: Electroencephalogram; ELISA: Enzyme-linked immunosorbent assay; EMG: Electromyogram; FAM: Fluorescein Phosphoramidite; FOSQ: Functional Outcome of Sleep Questionnaire; HIV: Human Immunodeficiency Virus; IFN-Y: Interferon-gamma; IL-6: Interleukin-6; IP-10/CXCL10: Interferon-gamma induced protein-10/C-X-C motif chemokine Ligand 10; LGTV: Langat Virus; MCP-1/CCL2:Monocyte Chemoattractant Protein-1/ C-C Motif Chemokine Ligand 2; NfL: Neurofilament light chain; NREM: NonRapid Eye Movement; PBS: Phosphate Buffered Saline; PC12 cells: Pheochromocytoma 12 cells; PCR: Polymerase Chain Reaction; pi: post infection; PSG: Polysomnography; RANTES/CCL5: Regulated on Activation, Normal T Expressed and Secreted/ C-C motif chemokine ligand 5; REM: Rapid Eye Movement; s.c.: subcutaneous; SARS-CoV-2: Severe Acute Respiratory Syndrome-Coronavirus type-2; SOL: Sleep Onset Latency; TBE: Tick-borne encephalitis; TBEV: Tick-borne encephalitis virus; TBEV-Eur: Tick-borne encephalitis virus European Subtype; TBEV-FE: Tick-borne encephalitis virus Far Eastern Subtype; TBEVSib: Tick-borne encephalitis virus Siberian Subtype

\section{Declarations}

\section{Ethical approval and consent to participate}

All animal studies were approved by the Animal Care and Experimentation Committee of the Canton of Bern, Switzerland (license no. BE 88/18).

\section{Consent for publication}

Not applicable

\section{Availability of the data and materials}

The datasets used and/or analyzed during the current study are available from the corresponding author on reasonable request.

\section{Competing interests}

The authors declare no competing interest

\section{Funding}

This project has been supported by the Interfaculty Research Cooperation Grant "Decoding Sleep" of the University of Bern, Switzerland, and additional support was provided by the Swiss National Science Foundation (grant 189136).

\section{Author contributions}

Author Contributions: GC, DG, SLL conceived the study. GC performed the experiments with the help of LGV and SS. GC, DG, and SLL analyzed the data and contributed to the writing of the Manuscript. GC, DG, 
AA, and SLL revised the manuscript for content.

\section{Acknowledgments}

We kindly thank Martin Palus and Daniel Růžek (Institute of Parasitology, Biology Centre of the Czech Academy of Sciences, Czech Republic) for providing the Langat Virus and Franziska Simon and Maria Erhardt for technical expertise.

\section{References}

1. Jarmer J, Zlatkovic J, Tsouchnikas G, Vratskikh O, Strauß J, Aberle JH, et al. Variation of the Specificity of the Human Antibody Responses after Tick-Borne Encephalitis Virus Infection and Vaccination. J Virol. American Society for Microbiology; 2014;88:13845-57.

2. Tonteri E, Kipar A, Voutilainen L, Vene S, Vaheri A, Vapalahti O, et al. The Three Subtypes of Tick-Borne Encephalitis Virus Induce Encephalitis in a Natural Host, the Bank Vole (Myodes glareolus). PLoS One. Public Library of Science; 2013;8:e81214.

3. Dörrbecker B, Dobler G, Spiegel M, Hufert FT. Tick-borne encephalitis virus and the immune response of the mammalian host. Travel Med Infect Dis. Elsevier; 2010;8:213-22.

4. Bogovic P, Strle F. Tick-borne encephalitis: A review of epidemiology, clinical characteristics, and management. http://www.wjgnet.com/. Baishideng Publishing Group Inc.; 2015;3:430-41.

5. Heinz FX, Stiasny K, Holzmann H, Grgic-Vitek M, Kriz B, Essl A, et al. Vaccination and Tick-borne Encephalitis, Central Europe - Volume 19, Number 1-January 2013 - Emerging Infectious Diseases journal - CDC. Emerg Infect Dis. 2013;19:69-76.

6. Süss J. Tick-borne encephalitis 2010: Epidemiology, risk areas, and virus strains in Europe and AsiaAn overview. Ticks Tick Borne Dis. Urban \& Fischer; 2011;2:2-15.

7. Factsheet about tick-borne encephalitis (TBE) [Internet]. [cited 2021 Jul 23]. Available from: https://www.ecdc.europa.eu/en/tick-borne-encephalitis/facts/factsheet

8. Mansfield KL, Johnson N, Phipps LP, Stephenson JR, Fooks AR, Solomon T. Tick-borne encephalitis virus - a review of an emerging zoonosis. J Gen Virol. Microbiology Society; 2009;90:1781-94.

9. Růžek D, Dobler G, Mantke OD. Tick-borne encephalitis: Pathogenesis and clinical implications. Travel Med Infect Dis. Elsevier; 2010;8:223-32.

10. Taba P, Schmutzhard E, Forsberg P, Lutsar I, Ljøstad U, Mygland Å, et al. EAN consensus review on prevention, diagnosis and management of tick-borne encephalitis. Eur J Neurol. John Wiley \& Sons, Ltd; 2017;24:1214-e61. 
11. Kaiser R. The clinical and epidemiological profile of tick-borne encephalitis in southern Germany 1994-98A prospective study of 656 patients. Brain. Oxford Academic; 1999;122:2067-78.

12. Mickienè A, Laiškonis A, Günther G, Vene S, Lundkvist Å, Lindquist L. Tickborne Encephalitis in an Area of High Endemicity in Lithuania: Disease Severity and Long-Term Prognosis. Clin Infect Dis. Oxford Academic; 2002;35:650-8.

13. Haglund M, Forsgren M, Lindh G, Lindquist L. A 10-Year Follow-Up Study of Tick-Borne Encephalitis in the Stockholm Area and a Review of the Literature: Need for a Vaccination Strategy. http://dx.doi.org/103109/00365549609027160. Taylor \& Francis; 2009;28:217-24.

14. Haglund M, Günther G. Tick-borne encephalitis-pathogenesis, clinical course and long-term followup. Vaccine. Elsevier; 2003;21:S11-8.

15. Misić Majerus L, Daković Rode O, Ruzić Sabljić E. [Post-encephalitic syndrome in patients with tickborne encephalitis]. Acta Med Croatica. 2009;63:269-78.

16. Czupryna P, Moniuszko A, Pancewicz SA, Grygorczuk S, Kondrusik M, Zajkowska J. Tick-borne encephalitis in Poland in years 1993-2008 - epidemiology and clinical presentation. A retrospective study of 687 patients. Eur J Neurol. John Wiley \& Sons, Ltd; 2011;18:673-9.

17. Czupryna P, Grygorczuk S, Krawczuk K, Pancewicz S, Zajkowska J, Dunaj J, et al. Sequelae of tickborne encephalitis in retrospective analysis of 1072 patients. Epidemiol Infect. Cambridge University Press; 2018;146:1663-70.

18. Veje M, Studahl M, Thunström E, Stentoft E, Nolskog P, Celik Y, et al. Sleep architecture, obstructive sleep apnea and functional outcomes in adults with a history of Tick-borne encephalitis. PLoS One. Public Library of Science; 2021;16:e0246767.

19. Veje M, Nolskog P, Petzold M, Bergström T, Lindén T, Peker Y, et al. Tick-Borne Encephalitis sequelae at long-term follow-up: a self-reported case-control study. Acta Neurol Scand. John Wiley \& Sons, Ltd; 2016;134:434-41.

20. Hidalgo H, Kallweit U, Mathis J, Bassetti CL. Post Tick-Borne Encephalitis Virus Vaccination Narcolepsy with Cataplexy. Sleep. Oxford Academic; 2016;39:1811-4.

21. Maffioli C, Grandgirard D, Engler O, Leib SL. A Tick-Borne Encephalitis Model in Infant Rats Infected With Langat Virus. J Neuropathol Exp Neurol. Oxford Academic; 2014;73:1107-15.

22. Maffioli C, Grandgirard D, Leib SL, Engler O. SiRNA Inhibits Replication of Langat Virus, a Member of the Tick-Borne Encephalitis Virus Complex in Organotypic Rat Brain Slices. PLoS One. Public Library of Science; 2012;7:e44703. 
23. Pace M, Adamantidis A, Facchin L, Bassetti C. Role of REM Sleep, Melanin Concentrating Hormone and Orexin/Hypocretin Systems in the Sleep Deprivation Pre-Ischemia. PLoS One. Public Library of Science; 2017;12:e0168430.

24. Bastianini S, Berteotti C, Gabrielli A, Del Vecchio F, Amici R, Alexandre C, et al. SCOPRISM: A new algorithm for automatic sleep scoring in mice. J Neurosci Methods. Elsevier; 2014;235:277-84.

25. Mensen A, Riedner B, Tononi G. Sleep Wave Analysis Toolbox for Matlab. 2015;

26. Lenz N, Engler O, Grandgirard D, Leib SL, Ackermann-Gäumann R. Evaluation of antivirals against tickborne encephalitis virus in organotypic brain slices of rat cerebellum. PLoS One. Public Library of Science; 2018;13:e0205294.

27. Disanto G, Barro C, Benkert P, Naegelin Y, Schädelin S, Giardiello A, et al. Serum Neurofilament light: A biomarker of neuronal damage in multiple sclerosis. Ann Neurol. John Wiley \& Sons, Ltd; 2017;81:85770.

28. Engman ML, Lindström K, Sallamba M, Hertz C, Sundberg B, Hansson MEA, et al. One-year follow-up of tick-borne central nervous system infections in childhood. Pediatr Infect Dis J. 2012;31:570-4.

29. Shen J, Barbera J, Shapiro CM. Distinguishing sleepiness and fatigue: Focus on definition and measurement. Sleep Med. Rev. W.B. Saunders; 2005. p. 63-76.

30. Kaiser R. Tick-borne encephalitis (TBE) in Germany and clinical course of the disease. Int J Med Microbiol. Urban \& Fischer; 2002;291:58-61.

31. Zawadzki R, Garkowski A, Kubas B, Zajkowska J, Hładuński M, Jurgilewicz D, et al. Evaluation of Imaging Methods in Tick-Borne Encephalitis. Polish J Radiol. Termedia; 2018;82:742-7.

32. Gent TC, Bassetti C LA, Adamantidis AR. Sleep-wake control and the thalamus. Curr Opin Neurobiol. Elsevier Current Trends; 2018;52:188-97.

33. Fuller P, Sherman D, Pedersen NP, Saper CB, Lu J. Reassessment of the structural basis of the ascending arousal system. J Comp Neurol. John Wiley \& Sons, Ltd; 2011;519:933-56.

34. Hermann DM, Siccoli M, Brugger P, Wachter K, Mathis J, Achermann P, et al. Evolution of Neurological, Neuropsychological and Sleep-Wake Disturbances After Paramedian Thalamic Stroke. Stroke. Lippincott Williams \& Wilkins; 2008;39:62-8.

35. Bassetti C, Marhis J, Gugger M, Lovblad KO, Hess CW. Hypersomnia following paramedian thalamic stroke: A report of 12 patients. Ann Neurol. John Wiley \& Sons, Ltd; 1996;39:471-80.

36. Veje M, Studahl M, Thunström E, Stentoft E, Nolskog P, Celik Y, et al. Sleep architecture, obstructive sleep apnea and functional outcomes in adults with a history of Tick-borne encephalitis. PLoS One. 
Public Library of Science; 2021;16.

37. Hutchison IC, Rathore S. The role of REM sleep theta activity in emotional memory. Front Psychol. Frontiers Media S.A.; 2015;6.

38. Holton CM, Hanley N, Shanks E, Oxley P, Mccarthy A, Eastwood BJ, et al. Longitudinal changes in EEG power, sleep cycles and behaviour in a tau model of neurodegeneration.

39. Matsuda Y, Ozawa N, Shinozaki T, Aoki K, Nihonmatsu-Kikuchi N, Shinba T, et al. Chronic antidepressant treatment rescues abnormally reduced REM sleep theta power in socially defeated rats. Sci Reports |. 123AD;11:16713.

40. Michlmayr D, Bardina S V., Rodriguez CA, Pletnev AG, Lim JK. Dual Function of Ccr5 during Langat Virus Encephalitis: Reduction in Neutrophil-Mediated Central Nervous System Inflammation and Increase in T Cell-Mediated Viral Clearance. J Immunol. American Association of Immunologists; 2016;196:4622-31.

41. Grygorczuk S, Osada J, Toczyłowski K, Sulik A, Czupryna P, Moniuszko-Malinowska A, et al. The lymphocyte populations and their migration into the central nervous system in tick-borne encephalitis. Ticks Tick Borne Dis. Urban \& Fischer; 2020;11:101467.

42. Grygorczuk S, Zajkowska J, Swierzbińska R, Pancewicz S, Kondrusik M, Hermanowska-Szpakowicz T. [Concentration of the beta-chemokine CCL5 (RANTES) in cerebrospinal fluid in patients with tick-borne encephalitis]. Neurol Neurochir Pol. 40:106-11.

43. Grygorczuk S, Osada J, Parczewski M, Moniuszko A, Świerzbińska R, Kondrusik M, et al. The expression of the chemokine receptor CCR5 in tick-borne encephalitis. J Neuroinflammation 2016131. BioMed Central; 2016;13:1-17.

44. Zhang X, Zheng Z, Liu X, Shu B, Mao P, Bai B, et al. Tick-borne encephalitis virus induces chemokine RANTES expression via activation of IRF-3 pathway. J Neuroinflammation. BioMed Central Ltd.; 2016;13.

45. Kułakowska A, Byfield FJ, Żendzian-Piotrowska M, Zajkowska JM, Drozdowski W, Mroczko B, et al. Increased levels of sphingosine-1-phosphate in cerebrospinal fluid of patients diagnosed with tick-borne encephalitis. J Neuroinflammation 2014 111. BioMed Central; 2014;11:1-9.

46. Kang X, Li Y, Wei J, Zhang Y, Bian C, Wang K, et al. Elevation of Matrix Metalloproteinase-9 Level in Cerebrospinal Fluid of Tick-Borne Encephalitis Patients Is Associated with IgG Extravassation and Disease Severity. PLoS One. Public Library of Science; 2013;8:e77427.

47. Ygberg S, Fowler $\AA$, Bogdanovic G, Wickström R. The Cerebrospinal Fluid Interleukin-6/Interleukin-10 Ratio Differentiates Pediatric Tick-borne Infections. Pediatr Infect Dis J. Lippincott Williams and Wilkins; 2020;39:239-43. 
48. Günther G, Haglund M, Lindquist L, Forsgren M, Andersson J, Andersson B, et al. Tick-borne encephalitis is associated with low levels of interleukin-10 in cerebrospinal fluid.

http://dx.doi.org/103402/iee.v1i06029. Taylor \& Francis; 2011;1:6029.

49. Kondrusik M, Zajkowska J, Pancewicz S, Swierzbińska R, Grygorczuk S, Hermanowska-Szpakowicz T. [Interferon gamma concentration in the cerebrospinal fluid of patients with tick-borne encephalitis]. Neurol Neurochir Pol. 2005;39:109-13.

50. Fowler A, Ygberg S, Bogdanovic G, Wickström R. Biomarkers in cerebrospinal fluid of children with tick-borne encephalitis: Association with long-term outcome. Pediatr Infect Dis J. Lippincott Williams and Wilkins; 2016;35:961-6.

51. Michałowska-Wender G, Losy J, Kondrusik M, Zajkowska J, Pancewicz S, Grygorczuk S, et al. [Evaluation of soluble platelet cell adhesion molecule sPECAM-1 and chemokine MCP-1 (CCL2) concentration in CSF of patients with tick-borne encephalitis]. Pol Merkur Lekarski. 2006;20:46-8.

52. Formanova PP, Palus M, Salat J, Hönig V, Stefanik M, Svoboda P, et al. Changes in cytokine and chemokine profiles in mouse serum and brain, and in human neural cells, upon tick-borne encephalitis virus infection. J Neuroinflammation 2019 161. BioMed Central; 2019;16:1-14.

53. Růžek D, Salát J, Singh SK, Kopecký J. Breakdown of the Blood-Brain Barrier during Tick-Borne Encephalitis in Mice Is Not Dependent on CD8+ T-Cells. PLoS One. Public Library of Science; 2011;6:e20472.

54. Zajkowska J, Moniuszko-Malinowska A, Pancewicz S, Muszyńska-Mazur A, Kondrusik M, Grygorczuk S, et al. Evaluation of CXCL10, CXCL11, CXCL12 and CXCL13 chemokines in serum and cerebrospinal fluid in patients with tick borne encephalitis (TBE). Adv Med Sci. Elsevier; 2011;56:311-7.

55. Huang W-Y, Huang C-C, Chang C-C, Kor C-T, Chen T-Y, Wu H-M. Associations of Self-Reported Sleep Quality with Circulating Interferon Gamma-Inducible Protein 10, Interleukin 6, and High-Sensitivity CReactive Protein in Healthy Menopausal Women. PLoS One. Public Library of Science; 2017;12.

56. Irwin MR, Olmstead R, Carroll JE. Sleep Disturbance, Sleep Duration, and Inflammation: A Systematic Review and Meta-Analysis of Cohort Studies and Experimental Sleep Deprivation. Biol Psychiatry. NIH Public Access; 2016;80:40.

57. Alirezaei Z, Pourhanifeh MH, Borran S, Nejati M, Mirzaei H, Hamblin MR. Neurofilament light chain as a biomarker, and correlation with magnetic resonance imaging in diagnosis of CNS-related disorders. Mol Neurobiol. NIH Public Access; 2020;57:469.

58. Tyrberg T, Nilsson S, Blennow K, Zetterberg H, Grahn A. Serum and cerebrospinal fluid neurofilament light chain in patients with central nervous system infections caused by varicella-zoster virus. J Neurovirol. Springer; 2020;26:719. 
59. Le ND, Muri L, Grandgirard D, Kuhle J, Leppert D, Leib SL. Evaluation of neurofilament light chain in the cerebrospinal fluid and blood as a biomarker for neuronal damage in experimental pneumococcal meningitis. J Neuroinflammation. BioMed Central; 2020;17.

60. Matsushige T, Ichiyama T, Kajimoto M, Okuda M, Fukunaga S, Furukawa S. Serial cerebrospinal fluid neurofilament concentrations in bacterial meningitis. J Neurol Sci. Elsevier; 2009;280:59-61.

61. Studahl M, Rosengren L, Günther G, Hagberg L. Difference in pathogenesis between herpes simplex virus type 1 encephalitis and tick-borne encephalitis demonstrated by means of cerebrospinal fluid markers of glial and neuronal destruction. J Neurol. J Neurol; 2000;247:636-42.

62. Kaiser R. Tick-borne encephalitis: Clinical findings and prognosis in adults. Wiener Medizinische Wochenschrift 2012 16211. Springer; 2012;162:239-43.

63. Gelpi E, Preusser M, Garzuly F, Holzmann H, Heinz FX, Budka H. Visualization of Central European Tick-Borne Encephalitis Infection in Fatal Human Cases. J Neuropathol Exp Neurol. Oxford Academic; 2005;64:506-12.

64. D’Angelo E. Physiology of the cerebellum. Handb Clin Neurol. Elsevier; 2018;154:85-108.

65. Hansson KE, Rosdahl A, Insulander M, Vene S, Lindquist L, Gredmark-Russ S, et al. Tick-borne Encephalitis Vaccine Failures: A 10-year Retrospective Study Supporting the Rationale for Adding an Extra Priming Dose in Individuals Starting at Age 50 Years. Clin Infect Dis. Oxford Academic; 2020;70:245-51.

66. Pentkowski NS, Rogge-Obando KK, Donaldson TN, Bouquin SJ, Clark BJ. Anxiety and Alzheimer's disease: Behavioral analysis and neural basis in rodent models of Alzheimer's-related neuropathology. Neurosci Biobehav Rev. Pergamon; 2021;127:647-58.

67. Krawczuk K, Czupryna P, Pancewicz S, Ołdak E, Moniuszko-Malinowska A. Comparison of tick-borne encephalitis between children and adults-analysis of 669 patients. J NeuroVirology 2020 264. Springer; 2020;26:565-71.

68. Barp N, Trentini A, Di Nuzzo M, Mondardini V, Francavilla E, Contini C. Clinical and laboratory findings in tick-borne encephalitis virus infection. Parasite Epidemiol Control. Elsevier; 2020;10:e00160.

69. Cornelius ADA, Hosseini S, Schreier S, Fritzsch D, Weichert L, Michaelsen-Preusse K, et al. Langat virus infection affects hippocampal neuron morphology and function in mice without disease signs. $J$ Neuroinflammation. BioMed Central; 2020;17.

70. Tesoriero C, Del Gallo F, Bentivoglio M. Sleep and brain infections. Brain Res Bull. Elsevier; 2019;145:59-74. 
71. Ibarra-Coronado EG, Pantaleón-Martínez AM, Velazquéz-Moctezuma J, Prospéro-García O, MéndezDíaz M, Pérez-Tapia M, et al. The Bidirectional Relationship between Sleep and Immunity against Infections. 2015;

72. Faraut B, Tonetti L, Malmartel A, Grabar S, Ghosn J, Viard J-P, et al. Sleep, Prospective Memory, and Immune Status among People Living with HIV. Int J Environ Res Public Health. Multidisciplinary Digital Publishing Institute (MDPI); 2021;18:1-15.

73. Heidbreder A, Sonnweber T, Stefani A, Ibrahim A, Cesari M, Bergmann M, et al. Videopolysomnographic findings after acute COVID-19: REM sleep without atonia as sign of CNS pathology? Sleep Med. Elsevier; 2021;80:92.

74. Hertenstein E, Gabryelska A, Spiegelhalder K, Nissen C, Johann AF, Umarova R, et al. Reference Data for Polysomnography-Measured and Subjective Sleep in Healthy Adults. J Clin Sleep Med. American Academy of Sleep Medicine; 2018;14:523.

75. Shevtsova AS, Motuzova O V., Kuragina VM, Akhmatova NK, Gmyl L V., Kondrat'eva Yl, et al. Lethal Experimental Tick-Borne Encephalitis Infection: Influence of Two Strains with Similar Virulence on the Immune Response. Front Microbiol. Frontiers Media SA; 2016;7:2172.

76. Eyer L, Kondo H, Zouharova D, Hirano M, Valdés JJ, Muto M, et al. Escape of Tick-Borne Flavivirus from 2'-C-Methylated Nucleoside Antivirals Is Mediated by a Single Conservative Mutation in NS5 That Has a Dramatic Effect on Viral Fitness. J Virol. American Society for Microbiology (ASM); 2017;91:102845 .

\section{Figures}


A

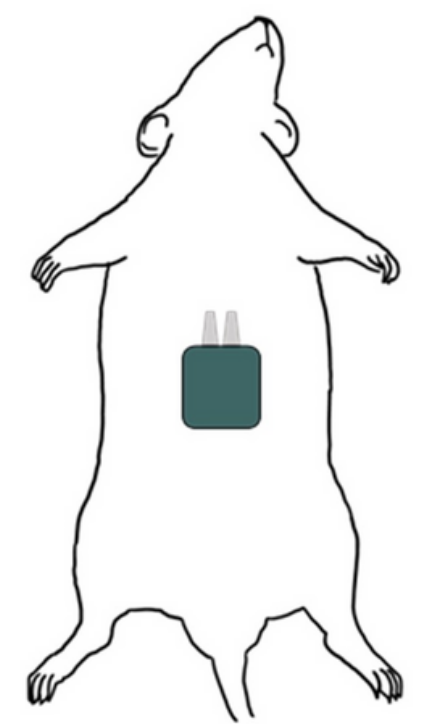

C

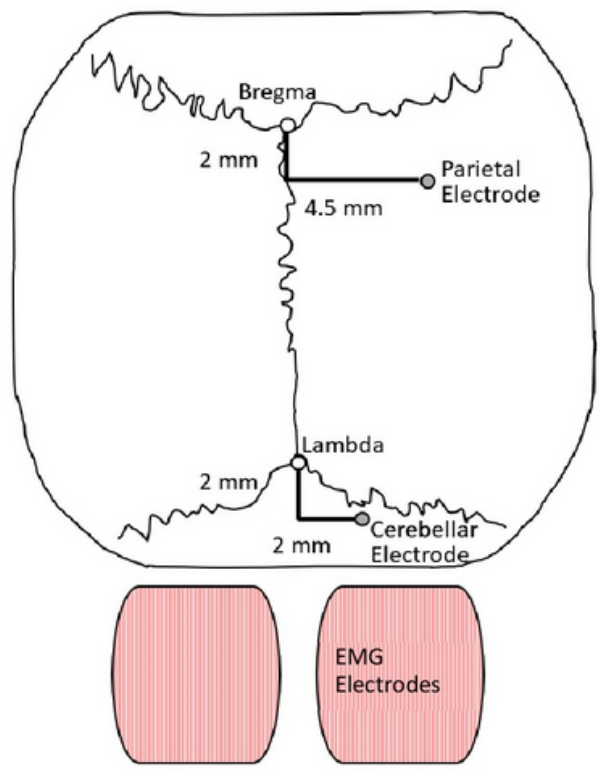

B

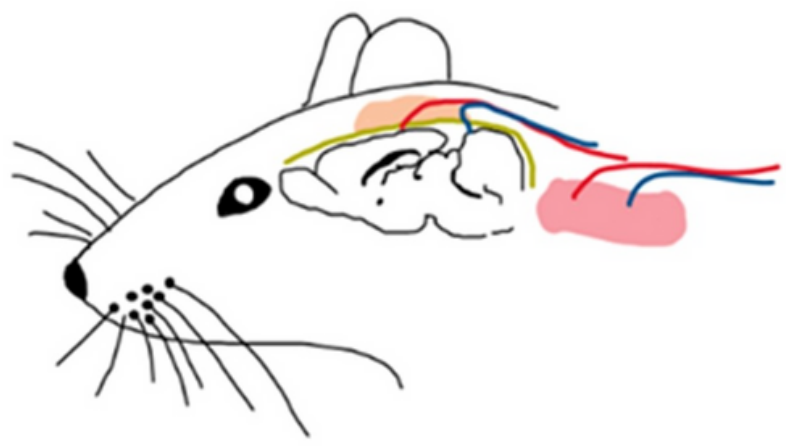

D

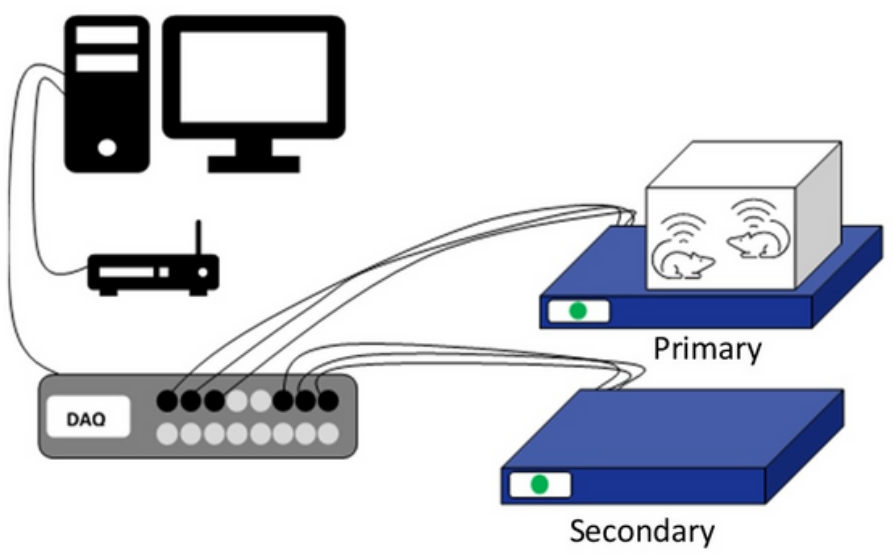

Figure 1

Experimental setting for Telemetry and EEG/EMG recording: (A) the placement of the telemetry body in the abdomen. (B) Representation of an animal head with implanted EEG and EMG electrodes. (C) Electrode (EEG and EMG) placement in the skull and neck muscle of the animal (not to scale). (D) Schematic displaying EEG/EMG recording. Two stacked SmartPads are used to allow Cohousing in a cage. The primary SmartPad is needed for wireless power to the implanted telemeters whereas each implanted sensor transmits the data separately through the two pads. 
A
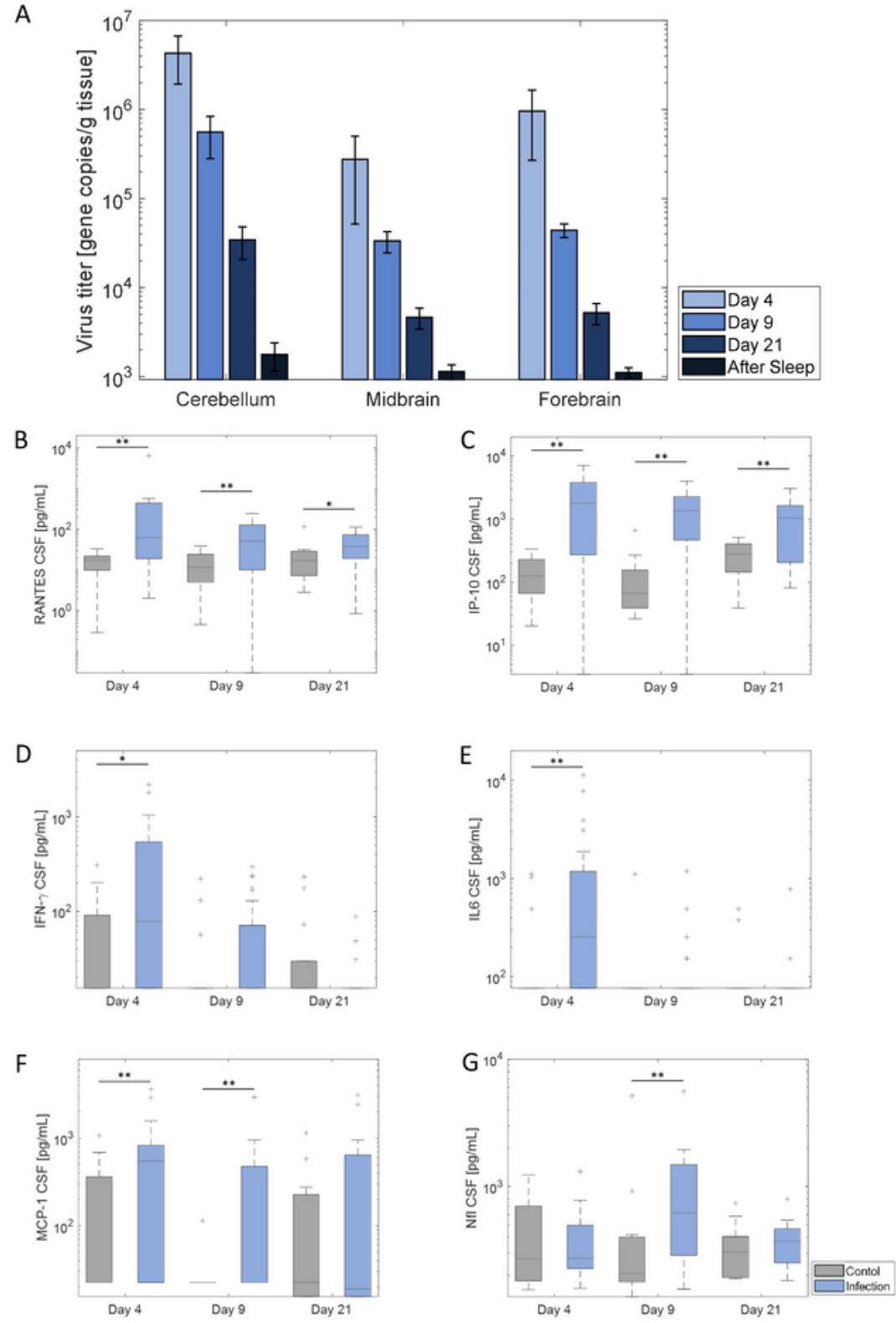

Figure 2

Virus load, Chemokines, Cytokines and NFL: (A) Virus titer at day 4 (ncerebellum $=11$; nmidbrain/forebrain=12), day 9 (ncerebellum=13; nmidbrain/forebrain=14), day $21(n=9)$ and after the sleep measurement $(n=6)$. (B-F) Chemokines and Cytokines assessed in the CSF of control and infected animals shown in. (G) Nfl level assessed in CSF in control and infected animals. $\dagger p<0.1, * p<0.05$, $* \star p$ $<0.01$ 

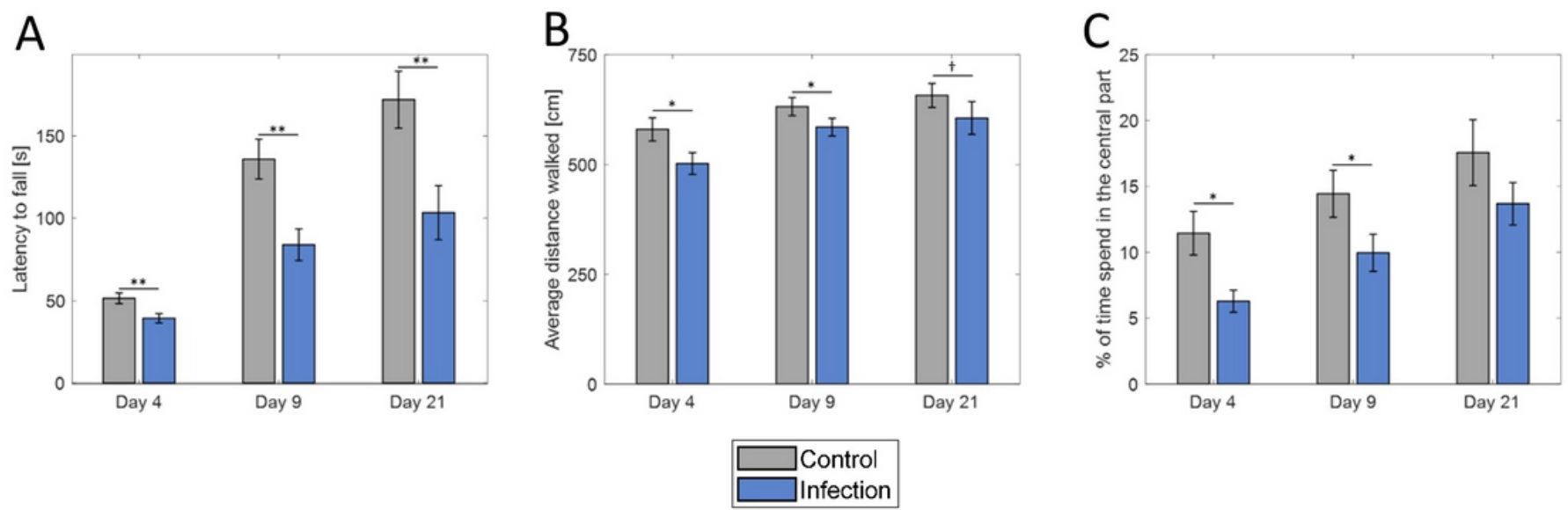

\section{Figure 3}

Behavioral tests: (A) Rotarod test showing the latency to fall in seconds (day 4: ncontrol=31, ninfection=36; day 9: ncontrol=22, ninfection=26; day 21 : ncontrol=15, ninfection=14). (B) and (C) Open field tests (day 4: $n=24$; day 9: $n=20$; day 21: ncontrol=13, ninfection=14) showing the average distance walked in $\mathrm{cm}$ and the percentage of the time spent in the center of the arena, respectively. $+p<0.1, * p<$ $0.05, * \star p<0.01$ 

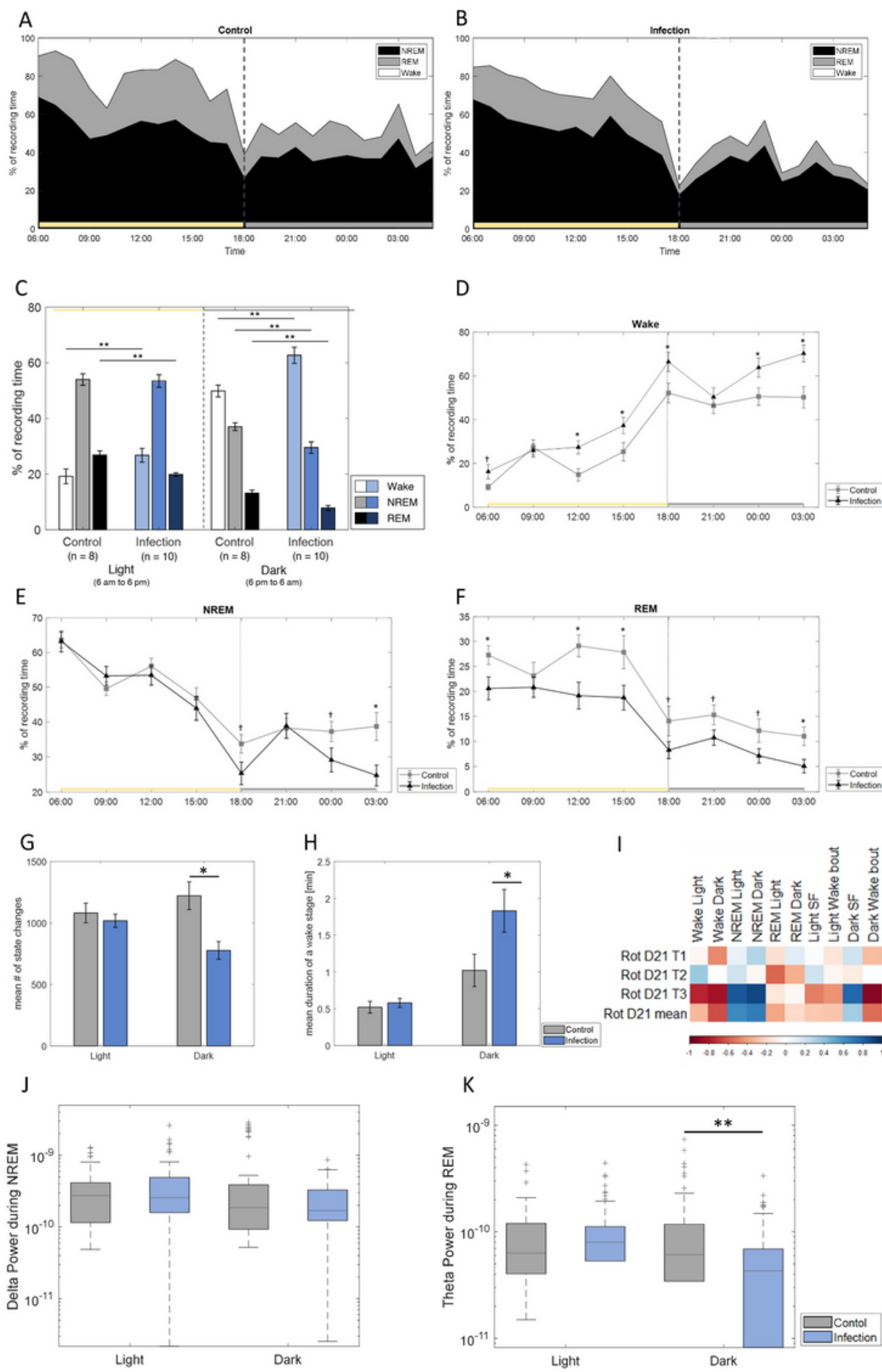

\section{Figure 4}

Sleep structure determination by EEG/EMG: Sleep macrostructure of control (A) and infected (B) animals over 24 hours. (C) Overview of the percentage in wake, NREM, and REM phases during light and dark period in control and infected animals. (D-F) Percentage of epochs displayed in 3 hours bins for the wake, NREM, and REM phases, respectively. (G) Mean number of state changes. $(H)$ Mean duration of wake stage. (I) Correlation between sleep markers and the rotarod performance at Day 21 in the infection 
group. (J) Delta power during NREM in the light and dark period. (K) Theta power during REM for the light and dark period. ncontrol $=8$, ninfection $=10 ; \uparrow p<0.1, * p<0.05$, ** $p<0.01$

\section{Supplementary Files}

This is a list of supplementary files associated with this preprint. Click to download.

- AdditionalFigure1.pdf

- AdditionalFigure2.pdf

- AdditionalFigure3.pdf

- AdditionalFigure4.pdf

- AdditionalTable1.pdf

- AdditionalTable2.pdf

- AdditionalTable3.pdf 